\title{
Modulation of Pimpinella tirupatiensis aqueous extract on Brain Mitochondrial Enzymes in STZ induced diabetic rats
}

\author{
D. Veera Nagendra Kumar ${ }^{1}$, K. R. Shanmugam ${ }^{2}$, Ch. Ramakrishna ${ }^{3}$, G. Venkata Subbaiah ${ }^{3}$, K. Sathyavelu Reddy ${ }^{3 *}$ \\ ${ }^{1}$ Division of Neuroscience, Department of Zoology, Govt. Degree College, Porumamilla, Andhra pradesh-516193, India. \\ ${ }^{2}$ Division of Ethnopharmacology, Department of Zoology, T.R.R. Government Degree College, Kandukur, A.P., India. \\ ${ }^{3}$ Division of Molecular Biology and Ethanopharmacology, Department of Zoology, Sri Venkateswara University, Tirupati - 517 502, India.
}

\begin{tabular}{l} 
ARTICLE INFO \\
\hline Article history: \\
Received on: $09 / 01 / 2017$ \\
Accepted on: $23 / 03 / 2017$ \\
Available online: $30 / 09 / 2017$ \\
\hline Key words: \\
Diabetes, Pimpinella \\
tirupatiensis, Mitochondrial \\
enzymes, Brain, Rats.
\end{tabular}

\begin{abstract}
The objective of the present study was to investigate the role of Pimpinella tirupatiensis $(\mathrm{Pt})$ aqueous extract on brain mitochondrial enzymes in Streptozotocin (STZ) induced diabetic rats. Adult male wistar strain rats were divided into five groups as NC (normal control), Pt. (Pimpinella treatment), DC (Diabetic control), D+Pt (Diabetic+ Pimpinella treatment), D+Gli (Diabetic + Glibenclamide treatment). Treatment was given as per the experimental protocol for 30days. Succinate dehydrogenase (SDH), glucose-6-phosphate dehydrogenase (G6PD), malate dehydrogenase (MDH), and glutamate dehydrogenase (GDH), and lactae dehydrogenase (LDH) enzymes activities were assayed in the brain tissue. In diabetic rats, we observed decreased activities of SDH, G6PDH, MDH, GDH and increased LDH activity. However with the Pimpinella tirupatiensis treatment mitochondrial enzymes activities were came back to normalcy. Our results suggest that the Pt supplementation modulates the mitochondrial enzymes activities in diabetic rats.
\end{abstract}

\section{INTRODUCTION}

Diabetes mellitus is a group of metabolic disorder characterized by abnormal elevated levels of blood glucose due to complete or relative insufficiency of insulin secretion as well as disturbances in carbohydrate, fat and protein metabolism. The neurological consequences of diabetes mellitus in the central nervous system (CNS) are now receiving greater attention. Glucose utilization is decreased in the brain during diabetes (McCall, 1992), providing a potential mechanism for increased vulnerability to acute pathological events. Reactive oxygen species (ROS) free radicals triggers neuronal disorders in diabetic condition. Pimpinella tirupatiensis is an endemic medicinal plant and constrained at seshachaa hills, tirupati, Andhra Pradesh. More recently, it was reported that Pimpinella

* Corresponding Author

K. Sathyavelu Reddy, Division of Molecular Biology and

Ethanopharmacology, Department of Zoology, Sri Venkateswara

University, Tirupati-517 502, India.Email: ksreddy2008@hotmail.com tirupatinsis is used to treat cough, stomach, asthma, ulcer and tooth ache (Madhava et al., 2008). Pimpinella tirupatiensis also possess antimicrobial, antioxidant properties. The current study was designed to explore the neuroprotective effect and role of $\mathrm{Pt}$ on mitochondrial enzymes in STZ induced diabetic rats.

\section{MATERIALS AND METHODS}

\section{Animals and treatment}

Wistar strain male albino rats, weighing $200 \pm 250 \mathrm{~g}$, were obtained from the Indian Institute of Science, Bangalore. The animals were housed in plastic cages and kept in the laboratory under constant temperature $\left(27 \pm 2^{\circ} \mathrm{C}\right)$ with 12 hrs light 12 hrs dark cycle. The rats were given a standard pellet diet and water ad libitum. The experiments were carried out in accordance with CPCSEA guidelines and the protocol was approved by the Institutional Animal Ethics Committee (regd. no. 438/01/a/ CPCSEA/ dt. 17.07.2001, in its resolution number 09 (iii)/a/CPSCA/IAEC/07-08/SVU/Zool/KSR-DVNK/dated 26/6/08. 


\section{Chemicals}

Streptozotocin (STZ) was purchased from Sigma (USA). All other chemicals and reagents used in this study were of analytical grade. obtained from the following scientific companies: Fisher (Pittsburgh, PA, USA), Merck (Mumbai, India), Ranbaxy (New Delhi, India) and Qualigens (Mumbai, India).

\section{Induction of diabetes}

The animals fasted over night and diabetes was induced by a single intra peritoneal injected with a freshly prepared STZ (40 mg/kg b.w) dissolved in ice cold $0.1 \mathrm{M}$ citrate buffer $(\mathrm{pH} 4.5)$ after allowing the rats for overnight fasting for 12-15 hr. $8 \mathrm{hr}$ after STZ administration the rats were kept for next $24 \mathrm{hr}$ on given $15 \%$ glucose solution to prevent hypoglycemia, as STZ is capable of producing fatal hypoglycemia due to destruction of $\beta$ cells which in turn results in to massive pancreatic insulin release. Diabetes was assessed by determining the fasting blood glucose after $48 \mathrm{hr}$ of injection of STZ. The blood glucose levels in STZ rats were increased to markedly higher levels than normal. After a week, when the condition of diabetes was stabilized, rats with marked hyperglycemia (blood glucose level $\geq 250 \mathrm{~g} / \mathrm{dl}$ ) were selected.

\section{Preparation of extract}

Tuberous roots of Pimpinella tirupatiensis $(\mathrm{Pt})$ were collected from Shesachalam hills, (Chittoor district, Andhra Pradesh, India) and identified by the Taxonomist of the Herbarium, Department of Botany, S. V. University, Tirupati. Voucher specimen (AECBT-05/2007-2008) was deposited in S. V. University Tirupati, Andhra Pradesh, India. Pimpinella tirupatiensis tuberous root was dried in the shade, powdered and the powder was used for the extraction of potential antidiabetic principles into water solvent. Pimpinella tirupatiensis tuberous root powder was soaked in water in different glass jars for 2 days at room temperature and the solvent was filtered. This was repeated three to four times until the extract give no coloration. The extract was distilled and concentrated under reduced pressure in the Rotary Evaporator (Model no-HS-2005V) and finally freeze dried by lyophilizer (Lyodel). The yield of the aqueous extract is $8.25 \%$ (w/w in terms of dried starting material) (Rajeswarareddy et al., 2012).

\section{Grouping of Animals}

The rats were divided into five groups, six rats in each group and treated as follows:

Group 1: Normal control (NC): this group of rats received vehicle solution ( $2 \%$ of Tween 80 ).

Group 2: Diabetic control (STZ $40 \mathrm{mg} / \mathrm{kg}$ body weight) (DC): streptozotocin is given intraperitoneally for the induction of diabetes to this group (Rajeswarareddy et al., 2012).

Group 3: Diabetic + glibenclamide treatment $(\mathrm{DC}+\mathrm{Gli})$ : diabetic rats received Pimpinella tirupatiensis aqueous extract $(750 \mathrm{mg} / \mathrm{kg})$ for a period of 30 days.

Group 4: Pimpinella tirupatiensis treatment (Pt.Aq.e): rats received aqueous extract of Pimpinella tirupatiensis with a dose of $750 \mathrm{mg} / \mathrm{kg}$ body weight for a period of 30 days (Rajeswarareddy et al., 2012)

Group 5: Diabetic + glibenclamide treatment $(\mathrm{D}+\mathrm{Gli})$ : diabetic rats treated with glibenclamide $(600 \mu \mathrm{g} / \mathrm{kg}$ body weight orally) (Shanmugam et al., 2011).

\section{Analytical procedures}

Blood samples were collected from all the rats before sacrifice and blood glucose levels were estimated using Glucometer (MediSense Optimum TMXceed, Abott Diabetes Care Inc., Almeda, USA). Oxidative enzymes like Succinate dehydrogenase (SDH), malate dehydrogenase (MDH) activity was estimated by the method of Nachlas et al. (1960). Glucose-6phosphate dehydrogenase (G6PDH) activity was assayed by the method of Lohr and Waller (1974). Glutamate dehydrogenase (GDH) was determined by the method of Lee and Lardy (1965). Lactae dehydrogenase (LDH) activity measured by Srikantan and Krishnamurti et al., (1955).

Completion of 30 days treatment, the animals sacrificed by cervical dislocation and the brain tissues were excised at $4^{0} \mathrm{C}$. The tissues were washed with ice-cold saline, immersed in liquid nitrogen and immediately stored at $-80{ }^{\circ} \mathrm{C}$ for further biochemical analysis.

The data were analyzed by using SPSS (Version 13.5; SPSS Inc., Chicago, IL, USA) and M.S. Office Excel Software for the significance of the main effects and along with their interactions. One way analysis of variance (ANOVA) was carried out with Dunnett's multiple comparison test and differences were considered significant at $P<0.01$.

\section{RESULTS AND DISCUSSION}

In this study, we reported increased levels of blood glucose in diabetic rats. However with Pimpinella tirupatiensis treatment significantly $(\mathrm{P}<0.01)$ decreased the blood glucose levels in diabetic group. Glibenclamide treatment also decreased the blood glucose levels in a significant manner when compared to diabetic group. The body weight of diabetic rats was also lower than the control group. However, Pimpinella tirupatiensis supplementation and glibenclamide treatment significantly improved the body weight and brought back towards near normal level.

Fig. 1-5 shows the effect of Pimpinella tirupatiensis aqueous extract on activities of brain mitochondrial enzymes in all the experimental groups. Our findings signify that in diabetic rats, G-6-PDH, SDH, MDH, GDH activities were significantly decreased. Whereas LDH activity was increased as compared to normal rats. Pimpinella tirupatiensis treatment significantly increase the G-6-PDH, SDH, MDH, GDH activities and decrease LDH activity as compared to diabetic rats. In the current study, we observed significant increase in blood glucose levels in diabetic rats (table 1). This may be due to the destruction of pancreatic $\beta$ cells by STZ, strengthening the hypothesis that STZ induces diabetes via the generation of free radicals. 
Table 1: Effect of oral administration of Pimpinella tirupatiensis on blood glucose in normal and diabetic control rats.

\begin{tabular}{|c|c|c|c|c|}
\hline \multirow[t]{2}{*}{ Groups } & \multicolumn{2}{|c|}{ Blood Glucose (mg/DL) } & \multicolumn{2}{|c|}{$\begin{array}{l}\text { Body Weights } \\
\text { (Grams) }\end{array}$} \\
\hline & 0 Day & 30 Day & 0 Day & 30 Day \\
\hline $\mathrm{NC}$ & $85 \pm 1.27$ & $96 \pm 2.5$ & $198 \pm 8.72$ & $209 \pm 12.56$ \\
\hline Pt.Aq.e & $88 \pm 1.52$ & $88 \pm 1.92$ & $192 \pm 2.42$ & $63 \pm 5.81$ \\
\hline $\mathrm{DC}$ & $257 \pm 2.94 *$ & $273 \pm 14.2^{*}$ & $195 \pm 2.96$ & $204 \pm 5.11$ \\
\hline DC+Pt.Aq.e & $263 \pm 3.97 *$ & $142 \pm 4.95^{*}$ & $197 \pm 5.89$ & $203 \pm 4.23$ \\
\hline $\mathrm{DC}+\mathrm{Gli}$ & $261 \pm 1.79 *$ & $93 \pm 2.89 *$ & $194 \pm 3.05$ & $206 \pm 3.94$ \\
\hline
\end{tabular}

All the values are \pm SD of six individual observations. Values are significant compared to diabetic control $(\mathrm{p}<0.001)$.

\begin{tabular}{|c|c|c|c|c|}
\hline aNC & aDC & QDC+PtAq.e & 口PtAq.e & aDC+Gli \\
\hline \multicolumn{5}{|c|}{ G6PDH } \\
\hline 8 & 0.35 & $a^{a}$ & a & $c$ \\
\hline छ & $\begin{array}{r}0.3 \\
0.25\end{array}$ & $b$ & & \\
\hline 赵 & 0.2 & $F$ & & \\
\hline 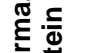 & 0.15 & & & \\
\hline 믄 흠 & 0.1 & & & \\
\hline 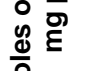 & $\begin{array}{r}0.05 \\
0\end{array}$ & & & \\
\hline $\begin{array}{l}\varepsilon \\
z\end{array}$ & & Treatm & & \\
\hline
\end{tabular}
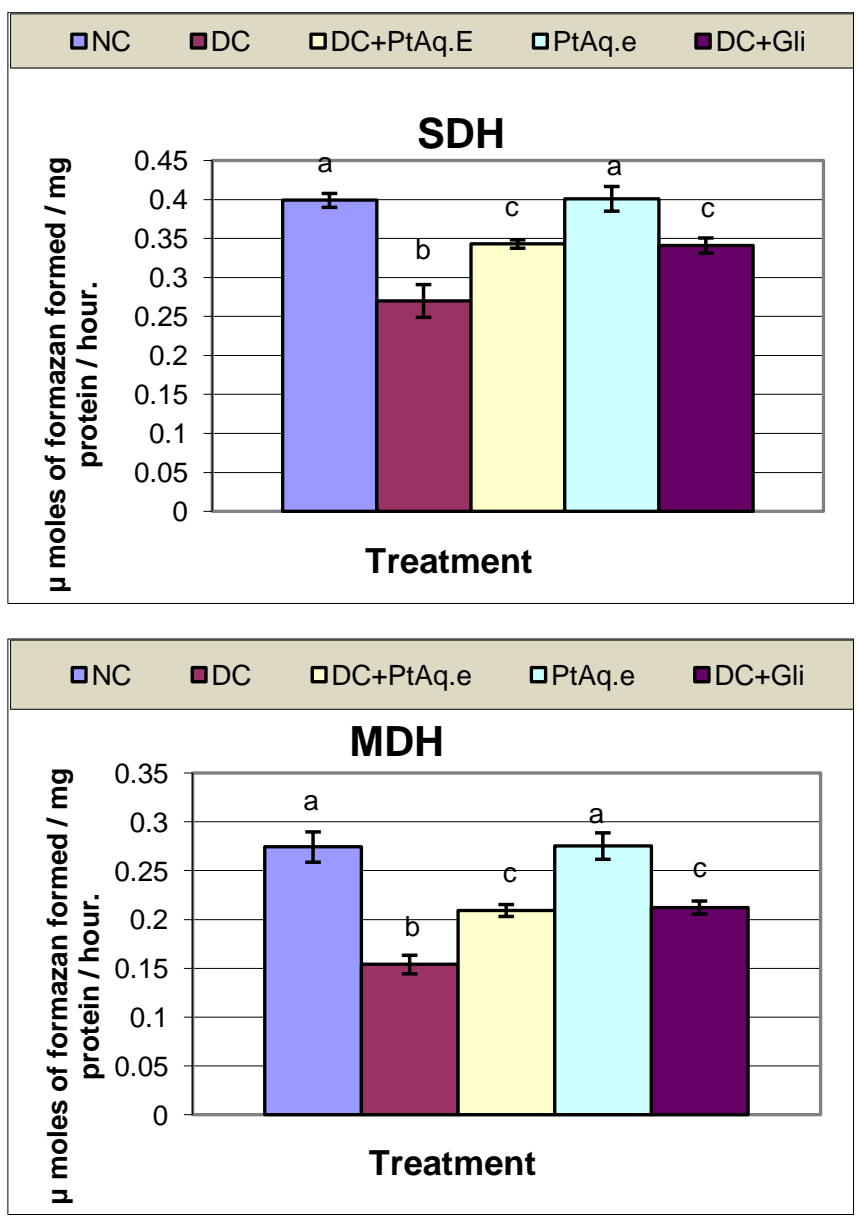

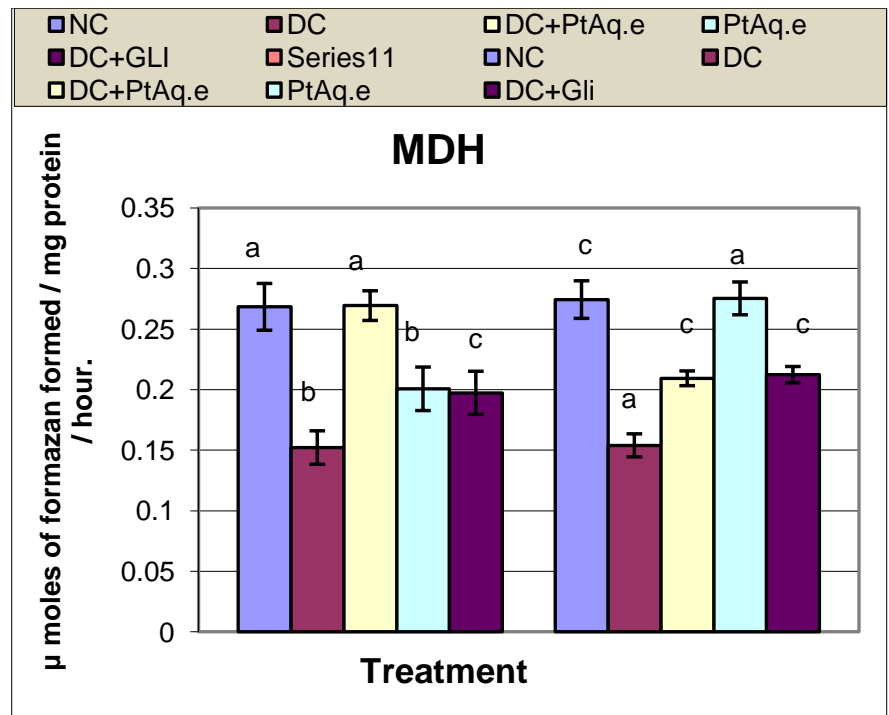

The increase in glucose levels in diabetic rats was due to an oxidative stress produced in the pancreas, due to a single strand break in pancreatic islets DNA. However with Pimpinella tirupatiensis treatment to STZ diabetic rats reduced blood glucose levels, in accordance with earlier reports (Dhanabal et al., 2006). In the current study, the blood glucose data clearly showed that Pimpinella tirupatiensis restrained the level of hyperglycemia resulting from the experimental destruction of beta pancreatic cells induced by STZ. The hypoglycemic effect of Pimpinella tirupatiensis increased gradually and was observed to be maximum at the end of the study period, i.e. 30 days. The decrease in blood glucose levels was due to the mechanism action of Pimpinella tirupatiensis. Our findings are similar to reported formerly for ginger (Shanmugam et al., 2011).

In the present study, a decrease in body weight in STZ induced diabetic rats. These results shows that the degradation of structural proteins is due to diabetes, and structural proteins are known to contribute to the body weight. However, with Pimpinella to diabetic rats, the body weights increased. This may be due to the antioxidant and hypoglycemic activity of Pimpinella tirupatiensis. But the body weight is not normalizing completely. SDH is a vital enzyme of citric acid cycle which catalyses the reversible oxidation of succinate to fumarate. In our study SDH activity was decreased in diabetic rats. This decrease would affect the conversion of succinate to fumarate and might cause a block in the Krebs cycle. It serves as a link between electron transport system and oxidative phosphorylation (Singer et al., 1972). The decrease in succinate dehydrogenase ( $\mathrm{SDH}$ ) activity due to oxidative stress condition indicates reduction in the conversion of succinate to fumarate resulting in depressed in oxidative metabolism mitochondria. During the diabetic stress condition diversion of phosphoenolpyruvate leads to increased formation of fumarate resulting in product inhibition of SDH (Moorthy et al., 1983), the decrease in the activities of SDH in tissues of diabetic rats can be associated with enzyme dysfunction due to activation of lipid 
peroxidation. In diabetic rats Pimpinella tirupatiensis treatment $S D H$ activity was increased when compared to diabetic rats. Shanmugam et al., (2011) reported that ginger treatment increased the SDH activity in diabetic rats. Increase in succinate dehydrogenase activity in Pimpinella tirupatiensis treated rats indicates better utilization of energy yielding intermediates by citric acid cycle.

G-6-PDH is a representative of the HMP shunt pathway and operates at the critical diversion point of the pentose phosphate pathway from glycolysis. In the present study, we observed decrease in the G6PDH in the brain tissue of diabetic rats. Similar to our findings, previous studies also demonstrated lower G6PD activity in diabetic tissues (Shanmugam et al., 2011). This suggested reduced conversion of glucose-6-phosphate dehydrogenase to 6-phosphogluconate leading to reduced formation NADPH and HMP shunt. This decrease may be considered as an adaptive phenomenon by which the supply of $\mathrm{NADPH}$ for aldose reductase is cut down, results are in consistence with earlier reports (Shanmugam et al., 2011; Rajeswarareddy et al., 2012). In the present study, the elevated G6PDH activity observed with the administration of Pimpinella tirupatiensis to diabetic rats may help reduce diabetes-associated complications. The Pimpinella tirupatiensis contain many antioxidant compounds, such as alkaloids, flavonols, flavones and volatile oils. These compounds may have antioxidant properties, with this property the extract could directly scavenges the free radicals.

In the current study $\mathrm{MDH}$ activity was decreased in diabetic rat brain tissue. In diabetic condition decrease of MDH suggests decreased utilization of malate. Previous studies (Shanmugam et al., 2011) have reported MDH activity was decreased in diabetic animal's tissues. This may be due to the disruption in TCA cycle, which leads to alterations in mitochondrial enzymes. Diabetes decreased the expression of genes involved in carbohydrate and energy metabolism through effects on known pathways such as glycolysis, citric acid cycle and oxidative phosphorylation. MDH activity was enhanced in diabetic rats with $P$. tirupatiensis treatment. This may be due to decreased oxidative stress and increased activities of mitochondrial enzymes. $\mathrm{Pt}$ has the capacity to increase the mitochondrial enzymes activities, this may due to the compounds which are present in $\mathrm{Pt}$ has the capacity to decrease the oxidative stress and increase these mitochondrial enzymes activities.

In this study, the activity of GDH was significantly decreased in the brain of diabetic rats. These results are in associated with the works of (Shanmugam et al., 2011; Nayeemunnisa et al., 2006). The decrease of GDH in the brain of rats due to activation of lipidperoxidation (Telushkin and Nozdrachev 1999), which attests to serious disturbances in energy metabolism and contributes to the impairment of glutamate utilization in the brain and progression of glutamate induced toxicity. In contrast, diabetic rats treated with Pimpinellaa tirupatiensis extract exhibited improvements in GDH activity. The increased levels may be due to the synchronization of energy metabolism and the elevation of glutamate levels in the cells. In diabetic rats with Pt treatment the GDH activity was increased. The increased activity might be due to the decreased activities of oxidative stress by Pimpinella tirupatiensis aqueous extract and increase the mitochondrial enzymes.

LDH plays an important role in carbohydrate metabolism and catalyses the inter-conversion of lactate and pyruvate. In the present study the brain LDH activity was significantly higher in diabetic rats. These results were similar to those of previous study that also demonstrated higher LDH activity in diabetic tissues (Shanmugam et al., 2011). Lactate dehydrogenase (LDH) is a key of anaerobic glycolysis and catalyses the reversible oxidation of lactate to pyruvate in the terminal step of glycolysis. The reaction catalyzed by lactate dehydrogenase interlinks anaerobic and aerobic oxidation. Excessive accumulation of pyruvate may result in higher LDH activity during diabetic condition. Excessive pyruvate is converted into lactate for which $\mathrm{LDH}$ is needed and, therefore, the activity of LDH may be increased due to less insulin availability in diabetes. The administration of $\mathrm{Pt}$ reduced the $\mathrm{LDH}$ activities in diabetic rats in a manner parallel to that induced by glibenclamide. The protective role of Pimpinella tirupatiensis. extract against these toxic effects indicates that this plant is able to prevent the harmful effects of diabetes. There are some reports on reversal of $\mathrm{LDH}$ in diabetic rats with treatment with ginger and Vitis vinifera. (Shanmugham et al., 2011, Giribabu et al., 2015). From the present study, it was concluded that $\mathrm{Pt}$ has neuroprotective and effect on mitochondrial enzymes in diabetic rat brain. Hence it may be used as neuroprotective agent against diabetic condition.

\section{Financial support and sponsorship: Nil.}

Conflict of interest: The authors declare no conflict of interest.

\section{REFERENCES}

Dhanabal SP, Kolkate CK, Ramanathan M, Kumar EP, Suresh B.. Hypoglycaemic activity of Pterocarpus marsupium Roxb. Phytother. Res 2006; 20: 4-8.

Donald Voet, Judith G Voet, Charlotte Pratt. Fundamentals of Biochemistry, 2nd Edition. John Wiley and Sons, Inc 2006; 547- 556.

Giribabu Nelli,Kilari Eswar Kumar,Somesula Swapna Rekha, Sekaran Muniandy, Naguib Salleh. Vitis vinifera (Muscat Variety) Seed Ethanolic Extract Preserves Activity Levels of Enzymes and Histology of the Liver in Adult Male Rats with Diabetes. Evidence-Based Complementary and Alternative Medicine 2015; 1-8.

Lee YP, Lardy HA. Influence of thyroid hormones on L- $\alpha$ glycerophosphate dehydrogenases and other dehydrogenases in various organs of the rat. J Biol Chem. 1965;240: 1427-1436.

Lohr GD, Waller HD. Glucose 6-phosphate dehydrogenase. In: Methods of enzymatic analysis. 2nd edition, New York, London: Academic Press, 1974; 2: 636-643.

Madhava Chetty K, Siraji K and Tulasi Rao K. Flowering Plants of Chittor District. Student Press, India 2008; 360-368.

McCall AL. The impact of diabetes on the CNS. Diabetes 1992; 41: $557-570$

Moorthy KS, Naidu MD, Chetty CS, Swami KS. Changes in carbohydrate metabolism in tissues of freshwater mussel (Lamellidensmarginalis) exposed to phosphamidon. Bull Environ Contam Toxicol 1983; 30:219-222. 
Nachlas MM, Margulies SI, Seligman AM. A colorimetric method for the estimation of succinic dehydrogenase activity. J Biol Chem 1960;235: 499-503.

Nayeemunnisa Nawaz R, Jayashree C. Changes in the regional protein metabolism in the central nervous system (CNS) of diabetic rats: Protection by Cichorium intybus (Chicory). Pharmacognosy Magazine 2006; 2: 130-132.

Rajeswarareddy S, Lavany T, Narasimhulu G, Sathyavelureddy K. Effect of Pimpinellatirupatiensison Oxidative Enzymes in STZ-induced Diabetic Rat Kidney. Iran J Pharm Res. 2012;11(1):277-86.

Shanmugam KR, Mallikarjuna K, Nishanth K, Li-Chen Lee, IShiung Cheng and Chia-Hua Kuo and Sathyavelu Reddy K. NephroProtective Effects of a Ginger Extract on Cytosolic and Mitochondrial Enzymes against Streptozotocin (STZ)-Induced Diabetic Complications in Rats. Chinese Journal of Physiology 2011; 54(2): 79-86.

Srikantan T, Krishnamurti C. Tetrazolium test for dehydrogenases. Journal of Scientific and Industrial Research. 1955;14: 206.

Singer TP, Kenney WC, Walker WH. Studies on Succinate dehydrogenase. XX. Amino acid sequence around the flavin site. J Biol Chem 1972; 247: 4510-3.

Telushkin PK, Nozdrachev AD. Hypoglycemia and the brain: metabolism and the mechanisms of neuronal damage. Usp.Fiziol. Nauk. 1999; 30: 14-27.

\section{How to cite this article:}

Nagendra Kumar DV, Shanmugam KR, Ramakrishna Ch., Subbaiah GV, Reddy KS. Modulation of Pimpinella tirupatiensis aqueous extract on Brain Mitochondrial Enzymes in STZ induced diabetic rats. J App Pharm Sci, 2017; 7 (09): 076-080. 